\title{
Rapamycin delays salivary gland atrophy following ductal ligation
}

\author{
SS Bozorgi ${ }^{1}$, GB Proctor ${ }^{1}$ and GH Carpenter ${ }^{*, 1}$
}

Salivary gland atrophy is a frequent consequence of head and neck cancer irradiation therapy but can potentially be regulated through the mammalian target of rapamycin (mTOR). Excretory duct ligation of the mouse submandibular gland provokes severe glandular atrophy causing activation of mTOR. This study aims to discover the effects of blocking mTOR signaling in ligation-induced atrophic salivary glands. Following 1 week of unilateral submandibular excretory duct ligation: gland weights were significantly reduced, 4E-BP1 and S6rp were activated, and tissue morphology revealed typical signs of atrophy. However, 3 days following ligation with rapamycin treatment, a selective mTOR inhibitor, gland weights were maintained, 4E-BP1 and S6rp phosphorylation was inhibited, and there were morphological signs of recovery from atrophy. However, following 5 and 7 days of ligation and rapamycin treatment, glands expressed active mTOR and showed signs of considerable atrophy. This evidence suggests that inhibition of mTOR by rapamycin delays ligation-induced atrophy of salivary glands.

Cell Death and Disease (2014) 5, e1146; doi:10.1038/cddis.2014.108; published online 20 March 2014

Subject Category: Experimental Medicine

Approximately 500000 people worldwide are diagnosed with head and neck cancer every year. ${ }^{1}$ Radiation therapy to the head and neck is a common treatment for such malignancies and salivary glands in the radiation field are severely damaged. Atrophy of the salivary glands is inevitable post radiation therapy and can also occur in autoimmune sialadenitis (Sjögren's syndrome) and obstructive sialadenitis. Patients experience reduced salivary flow, xerostomia, dental caries, mucosal infection, dysphagia, considerable discomfort and pain. ${ }^{2,3}$

Salivary gland atrophy can be recreated experimentally in rodents via ligation of the main excretory duct of the submandibular gland, which creates a histological appearance comparable with that which occurs in humans, ${ }^{4}$ which involves deletion of acinar cells through apoptosis ${ }^{5}$ and autophagy, ${ }^{6}$ as well as mitotic proliferation of ductal cells. ${ }^{5}$

One potentially important mechanism of regulating atrophy in salivary gland and other tissues is through the mammalian target of rapamycin; ${ }^{7}$ a highly conserved serine/threonine protein kinase which integrates cues from nutrients and growth factors, acting as a nexus point for cellular signals to control growth, metabolism and longevity. Activated mTOR regulates protein synthesis by phosphorylating ribosomal S6 kinase 1 (S6K1) and eukaryotic translation initiation factor $4 \mathrm{E}$-binding protein $1(4 \mathrm{E}-\mathrm{BP} 1)^{8}$ at multiple sites. ${ }^{9}$ Although in normal circumstances, mTOR is switched on in some tissues such as muscle and liver, it is normally switched off in salivary glands. mTOR is potentially instrumental in controlling acinar and ductal atrophy as it gets switched on after duct ligationinduced atrophy in the animal model, ${ }^{10}$ however the role of
mTOR in ligation-induced atrophy of salivary glands is still not fully clear.

Rapamycin is a specific inhibitor of mTOR signaling that binds directly to the mTOR complex 1 (mTORC1) and suppresses mTOR-mediated phosphorylation of S6K1 and $4 \mathrm{E}-\mathrm{BP} 1 .^{8}$

In the present study, rapamycin was used to study the effects of mTOR inhibition on duct ligation-induced salivary gland atrophy.

\section{Results}

Gland weights. Mean submandibular gland weight was significantly reduced in the 3 day ligation group (0.040 $\pm 0.001 \mathrm{~g}, n=3$ ), compared with nonligated control mice $(0.055 \pm 0.003 \mathrm{~g}, n=4)$. Following rapamycin treatment $(0.056 \pm 0.002 \mathrm{~g}, n=4)$, mean gland weight was significantly $(P=0.0008)$ greater compared with the ligation only group and was not different to unoperated controls. However, 5 day $(0.040 \pm 0.005 \mathrm{~g}, n=3)$ and 7 day ligation $(0.035 \pm 0.003 \mathrm{~g}$, $n=4$ ) groups experienced a significant reduction compared with unoperated controls, which was not affected by rapamycin treatment (Figure 1).

All control groups showed similar gland weight measurements, with unoperated controls, rapamycin only and rapamycin vehicle-treated groups all showing no statistically significant difference.

By the end of the experiment, there was no statistically significant difference in body weight between experimental mice and the controls. For example, neither the 7 day ligation

\footnotetext{
${ }^{1}$ King's College London Dental Institute, Salivary Research Unit, Floor 17, Tower Wing, London, UK

*Corresponding author: GH Carpenter, King's College London Dental Institute, Salivary Research Unit, Floor 17, Tower Wing, London SE1 9RT, UK. Tel: +44 207188 7460; Fax: +44 207188 7450; E-mail: guy.carpenter@kcl.ac.uk

Keywords: mTOR; rapamycin; atrophy; submandibular gland; ligation

Abbreviations: 4E-BP1, eukaryotic translation initiation factor 4E-binding protein 1; S6K1, ribosomal S6 kinase 1; S6rp, S6 ribosomal protein; mTOR, mammalian target of rapamycin; mTORC1, mammalian target of rapamycin complex1; mTORC2, mammalian target of rapamycin complex2; PI3K, phosphatidylinositide 3-kinase; PAS, periodic acid-Schiff's; AB/PAS, alcian blue/periodic acid-Schiff's; DMAB, p-dimethylaminobenzaldehyde; H\&E, hematoxylin and eosin

Received 22.11.13; revised 13.2.14; accepted 14.2.14; Edited by A Stephanou
} 


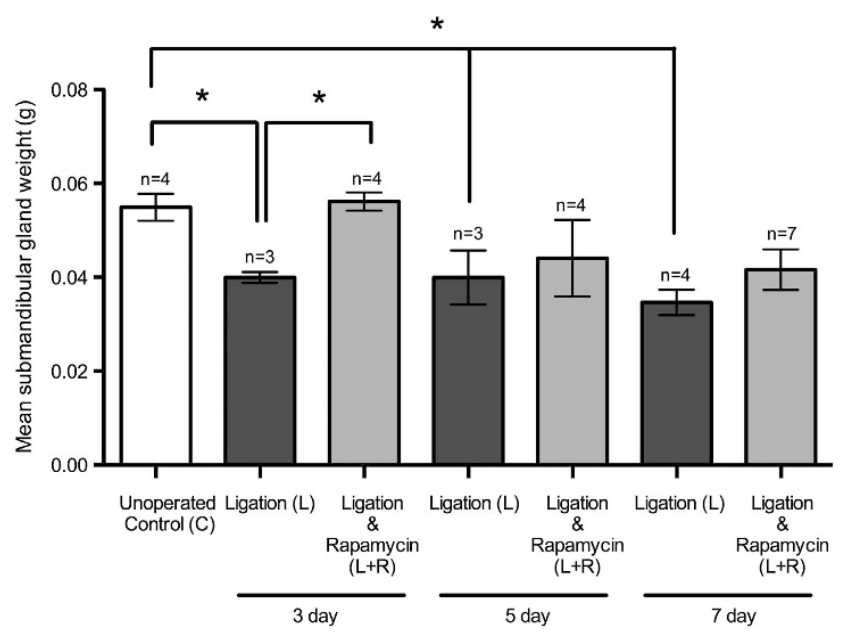

Figure 1 Submandibular gland weights. Mean submandibular gland weights were significantly $\left({ }^{*} P<0.05\right.$ ) reduced at 3,5 and 7 day ligation only groups in comparison with unoperated controls. Post ligation surgery and 3 days of rapamycin treatment, the gland weights increased $40 \%$ greater than the ligation only group. Five days and seven days of rapamycin treatment post ligation did not significantly alter gland weights in comparison with their respective ligation only groups. Data is expressed as mean \pm S.E.M.

only (28.52 $\pm 0.80 \mathrm{~g}, n=4)$ nor 7 day ligation with rapamycin treatment $(31.47 \pm 1.18 \mathrm{~g}, \quad n=7)$ differed significantly $(P=0.22)$ from unoperated control $(31.20 \pm 1.49 \mathrm{~g}, n=4)$ mice body weights.

\section{mTOR status}

Complete inhibition of mTOR after 3 days of rapamycin treatment: Immunoblotting of phospho-S6 ribosomal protein, a downstream component of the mTOR substrate S6K1 in glandular homogenates revealed activation of S6K1 (and therefore activation of mTOR) in only the ligated state in the 3 day group. However, rapamycin treatment abolished this activation (Figure $2 \mathrm{a}$ and quantified in Figure $2 \mathrm{c}$ ).

Low expression of 4E-BP1 occurred, as the inactive isoform, in unoperated controls. Ligation revealed an increase in 4E-BP1 phosphorylation (and therefore activation of mTOR) in 3 day groups, as shown by the presence of a higher molecular weight band. Rapamycin treatment following ductal ligation abolished this activation, as represented by a visible reduction of isoforms (Figure $2 \mathrm{~b}$ and quantified in Figure 2c). Rapamycin only treated glands showed no expression of mTOR activity, identical to unoperated controls.

Incomplete inhibition of mTOR during long term rapamycin treatment: Immunoprobing of phospho-S6 ribosomal protein expression on 3,5 and 7 day samples indicated mTOR activation in the ligated state. However, whilst rapamycin treatment abolished this activation after 3 days; a small protein band remained visible following rapamycin treatment after longer periods of ductal ligation (Figure $3 a$ and quantified in Figures $3 c$ and d).

Duct ligation at 5 and 7 days increased 4E-BP1 protein expression and increased phosphorylation status compared with control, suggesting mTOR activation. Following ligation and rapamycin treatment for 5 days glandular homogenates revealed incomplete reduction of hyperphosphorylated status in all four mice examined. Seven day glandular homogenates also showed a visible increase in 4E-BP1 in ligation and a reduction of all isoforms post rapamycin treatment, however the upper isoform was only slightly reduced following rapamycin treatment (Figure $3 \mathrm{~b}$ and quantified in Figures $3 \mathrm{c}$ and d). Rapamycin only treated glands showed no expression of mTOR substrate activity, identical to unoperated controls.

Western blotting of total mTOR in submandibular glands (Figure $3 e$ and quantified in Figure 3f) showed a marked increase in mTOR presence in ligated glands at all time points in comparison with controls. Rapamycin treatment greatly reduced total mTOR protein expression, but did not completely inhibit the presence of mTOR.

Short term rapamycin treatment rescues acinar atrophy: Periodic acid-Schiff's (PAS) staining of glandular homogenates indicated significant loss of acinar mucin with ligation at all time points (Figure 4). The 3 day ligation with rapamycin group showed only partial loss of mucin. Whereas 7 day rapamycin and ligation groups experienced complete loss of mucin, even post rapamycin treatment (Figure 4a). Five day groups experienced variable results with partial loss of mucin in some animals (Figure $4 \mathrm{~b}$ ) and a complete loss in other animals.

Histological assessments: The haematoxylin and eosin staining (H\&E) of both the ligation (Figure $5 \mathrm{~b}$ ) and the ligation with rapamycin treatment (Figure 5c) groups showed the presence of inflammatory cell infiltration, which were composed mainly of neutrophils and macrophages (as previously mentioned $)^{10,11}$ in the connective tissue between the lobules and among the parenchymal elements after 3 days in comparison with controls (Figure 5a). Three day ligation groups also revealed shrunken acinar cells with loss of secretory granules and duct luminal dilation as they underwent degranulation, similar to previous findings. ${ }^{12}$ Rapamycin-treated ligated glands didn't appear to affect inflammatory cell infiltrates, but showed intact acinar cells and some larger than normal acinar cells.

Morphometric analysis of H\&E-stained samples revealed the mean area \pm Standard Error of the Mean (S.E.M.) of the acini from the 3 day ligation samples $\left(227.40 \pm 30.72 \mu \mathrm{m}^{2}\right.$, $n=60)$ significantly decreased $(P<0.0001)$ in comparison with the control glands $\left(469.90 \pm 17.35 \mu \mathrm{m}^{2}, n=80\right)$. Rapamycin treatment following ligation rescued the acini size $\left(541.90 \pm 47.56 \mu \mathrm{m}^{2}, n=80\right)$ significantly $(P<0.0001)$ from the ligated state (Figure $5 \mathrm{~d}$ ).

Histological assessments of alcian blue/periodic acidSchiff's (AB/PAS)-stained adult female submandibular gland sections after 3 day rapamycin treatment following ductal ligation (Figure $6 \mathrm{~b}$ ) revealed enlarged acinar cells as shown in H\&E staining that are strongly stained by alcian blue, when compared with ligation only (Figure 6a).

The H\&E staining of the 7 days ligation and the 7 days ligation with rapamycin treatment, the 5 days ligation and the 5 days ligation with rapamycin treatment groups all revealed very similar results as ligation only glands with very little, to no differences.

Histomorphometric analysis indicated that the area of acini from ligated glands was significantly reduced at longer time 


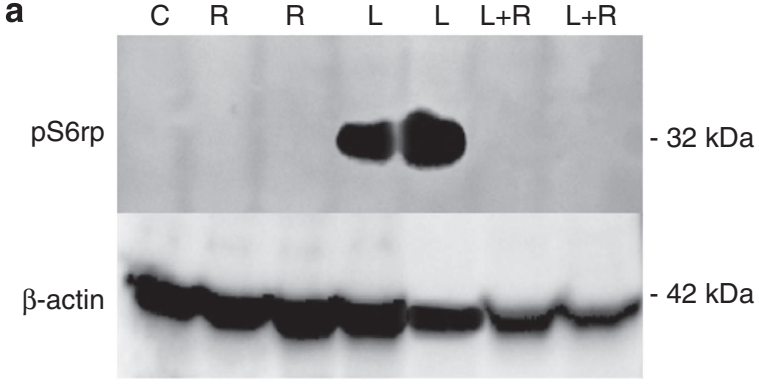

b
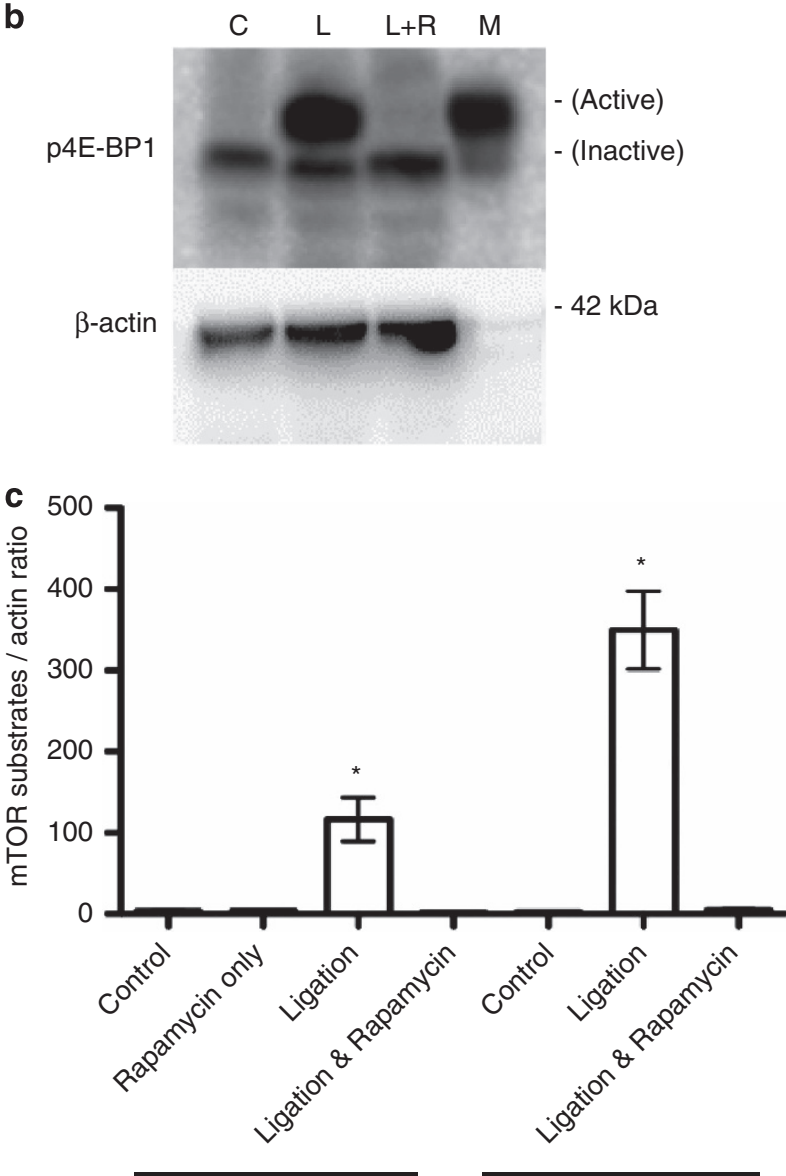

Phospho-S6rp

Phospho-4E-BP1

Figure 2 Immunoblotting of phospho-S6 ribosomal protein (pS6rp) (a) and phospho-4E-BP1 protein (b) expression in submandibular glands of 3 day groups. pS6rp showed no expression in controls (c) and rapamycin only (R). Presence of pS6rp indicates activation of mTOR in the ligated state $(L)$ but rapamycin treatment post ligation $(\mathrm{L}+\mathrm{R})$ abolished this activation. Phospho-4E-BP1 protein expression in controls (c) showed an inactive isoform of $4 \mathrm{E}-\mathrm{BP} 1$, whereas ligation $(\mathrm{L})$ increased 4E-BP1 phosphorylation and therefore activation, indicating mTOR phosphorylation. Rapamycin treatment following ductal ligation $(L+R)$ abolished this activation, as represented by a visible reduction back to the inactive isoform. Muscle homogenates (M) were used as a positive control. Beta actin ( $\beta$-actin) was used as a loading control, however muscle homogenates showed absence of beta actin ( $\beta$-actin) as muscle expresses $\alpha$-smooth muscle actin ( $\alpha$-SMA). Densitometric analysis from the 3 day experiments (c) show pS6rp and p4E-BP1 phosphorylation as a ratio of $\beta$-actin, ${ }^{*} P<0.05$ in comparison with controls. The bars represent the mean \pm S.E.M., ANOVA $\left({ }^{*} P<0.05\right)$ data represents results from at least three different experiments

periods, in both ligated and rapamycin-treated ligated samples, compared with unoperated control samples.

The histochemical staining of granular ducts by DMABnitrite showed loss of kallikrein, which is a marker for ductal function, ${ }^{13}$ secretory granules of granular tubule cells 3 days post duct ligation (Figure 6d) in comparison with unoperated controls (Figure $6 \mathrm{c}$ ), rapamycin treatment following ligation did not rescue the reduction of stored tissue secretory granules containing kallikreins (Figure 6e). All 5 and 7 day experimental groups (ligation only and the ligation with rapamycin groups) indicated a complete loss of DMAB in comparison with unoperated controls.

\section{Discussion}

Our previous study suggested that mTOR was associated with the atrophic process during submandibular duct ligation. This study provides further evidence that mTOR is required for an autophagy-like process. However this study underlines the complexity of the in vivo regulation of mTOR and hints at its interaction with other pathways.

The ligation of the main excretory duct of the submandibular gland to study atrophy of the salivary glands has been well characterized in rats. ${ }^{14,15}$

As the first study of it's kind to use mice, this study found that the ligation of the excretory duct of the submandibular gland led to glandular atrophy as the gland underwent morphological, cellular and microscopic changes.

One such change is that mean submandibular gland weight was significantly reduced in all ligation groups in comparison with controls. Decreased volume and size of acinar cells with acinar and ductal degranulation may explain the significant decrease of glandular weights, although the increase of inflammatory cells infiltrating may have added to the gland weight initially.

Tissue morphology indicated that the glands of the 3 day ductal ligation group had shrunken acinar cells with loss of secretory granules, duct luminal dilation as they underwent degranulation and a general loss of cytoplasm in the duct cells. Similarly at 5 and 7 days of ligation most acinar cells had disappeared, ductal lumena were dilated with little cytoplasm left and there was an increased amount of connective tissue filled with inflammatory cell infiltrates.

The DMAB staining specific for stored tissue kallikrein of the granular convoluted ducts ${ }^{16}$ of submandibular gland tissues at all time points post ligation indicated loss of kallikreincontaining secretory granules, corresponding with PAS staining of glandular homogenates, which showed a complete loss of mucin with ligation as previously demonstrated in ductligated submandibular gland of rats. ${ }^{17}$ The absence of secretory glycoproteins indicates a lack of acinar cell synthetic activity.

Immunoprobing of the phospho-S6 ribosomal protein, which is phosphorylated at several sites by S6K1, ${ }^{18,19}$ and 4E-BP1 (another mTORC1 substrate) confirmed that mTOR is activated during ligation-induced atrophy of the salivary glands, which corresponds with the start of autophagic processes during ligation-induced atrophy. ${ }^{10}$

Three days of rapamycin treatment following duct ligation showed a complete inhibition of mTOR, as shown by 

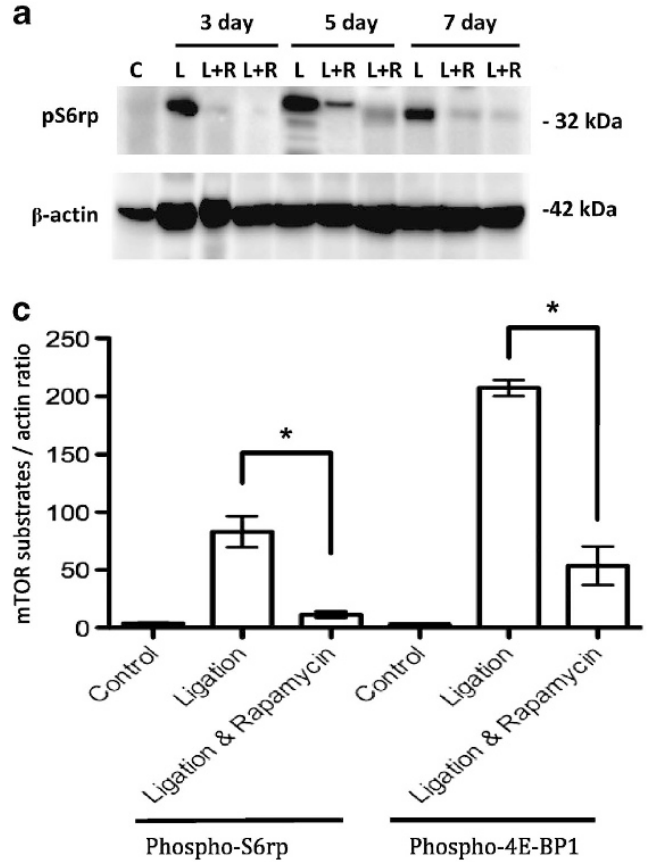

e

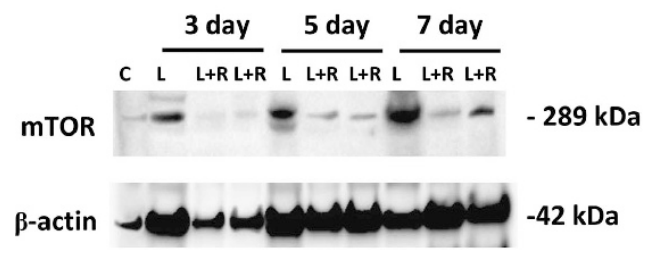

b
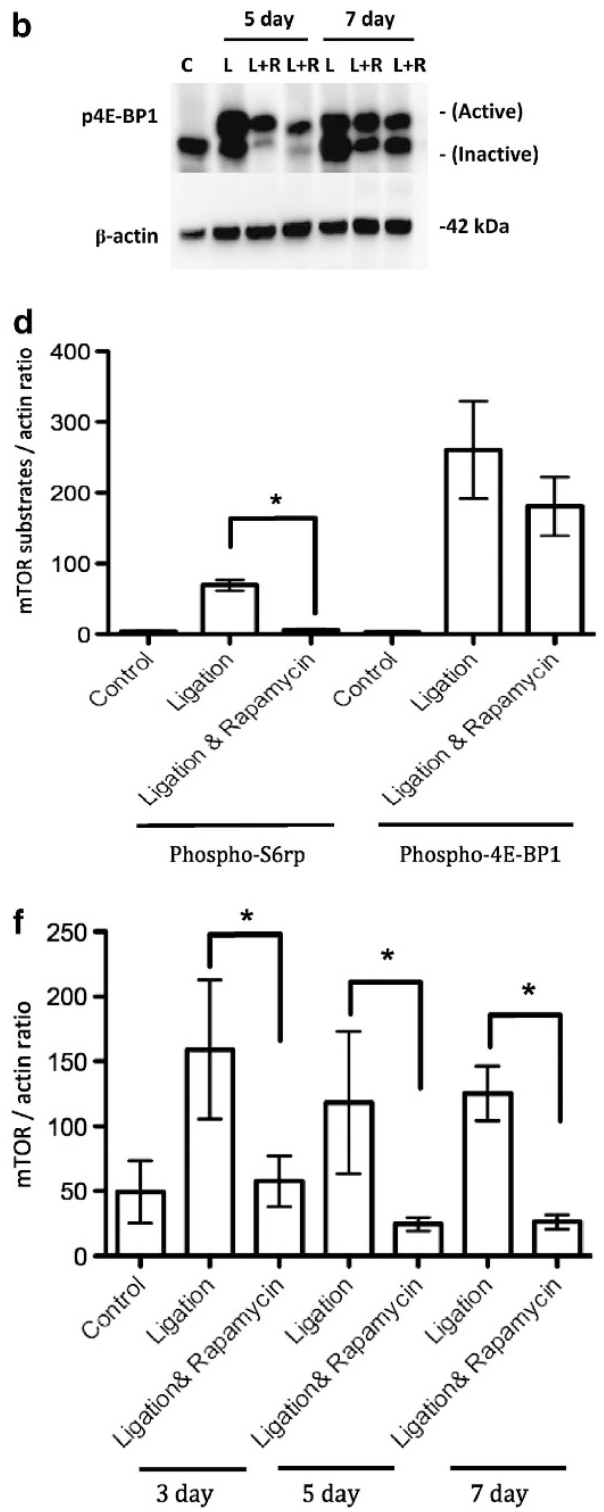

Figure 3 Immunoblotting of phospho-S6 ribosomal protein (pS6rp) (a) and phospho-4E-BP1 protein (b) expression in submandibular glands during the longer 5 and 7 day periods of ligation and ligation with rapamycin treatment. pS6rp expression was abolished after 3 day rapamycin treatment post ductal ligation $(L+R)$ hence inhibiting $m T O R$ expression. Five and seven day ligated (L) glandular homogenates revealed increased pS6r-protein expression. Rapamycin treatment during ductal ligation showed a visible reduction but not complete abolishment of expression at 5 and 7 days, indicating incomplete inhibition of mTOR. Phospho-4E-BP1 protein expression experienced a marked increase (the activated isoform bands), after ligation (L) for 5 and 7 days, indicating phosphorylation of mTOR. Rapamycin treatment following ductal ligation ( $L+R$ ) on 5 day showed a visible reduction of isoforms but not complete return to inactive isoform bands, indicating incomplete inhibition of mTOR, whereas 7 day ligation with rapamycin treatment revealed no visible reduction of 4E-BP1 protein expression. Densitometric analysis from the 5 (c) and 7 day experiments (d) show pS6rp and p4E-BP1 phosphorylation as a ratio of $\beta$-actin $\left({ }^{\star} P<0.05\right)$. The bars represent the mean \pm S.E.M. Protein expression of total $\mathrm{mTOR}$ in submandibular glands (e) in unoperated control, ligated and ligated with rapamycin-treated mice at increasing time points. Rapamycin treatment reduces total mTOR protein expression but does not completely inhibit mTOR, correlating with the pS6rp and 4E-BP1 protein expressions. Densitometric analysis showing mTOR expression as a ratio of $\beta$-actin $(\mathbf{f})\left({ }^{*} P<0.05\right)$. The bars represent the mean \pm S.E.M. Beta actin ( $\beta$-actin) was used as a loading control. Data represents results from at least three independent experiments

the immunoprobing of mTOR substrates S6rp and 4E-BP1. Tissue morphology revealed intact acinar cells, although the ducts displayed larger lumena compared with control mice with the presence of inflammatory cells suggesting ductal atrophy. The preservation of mucincontent post treatment suggests that rapamycin maintains synthesis or prevents degradation of secretory glycoproteins by fully inhibiting the activity of mTOR. Therefore, inhibition of mTOR can delay ligation-induced atrophy of salivary glands, however only affecting acinar, but not ductal, atrophic processes.

However, longer periods of rapamycin treatment post ligation surgery showed a loss of efficacy as gland weights were reduced, with morphological changes similar to ligation only and phosphorylation of S6rp and 4E-BP1 showing an incomplete inhibition of mTOR. Based on the results obtained in this experiment, rapamycin treatment is not believed to be effective in longer periods of administration and that 

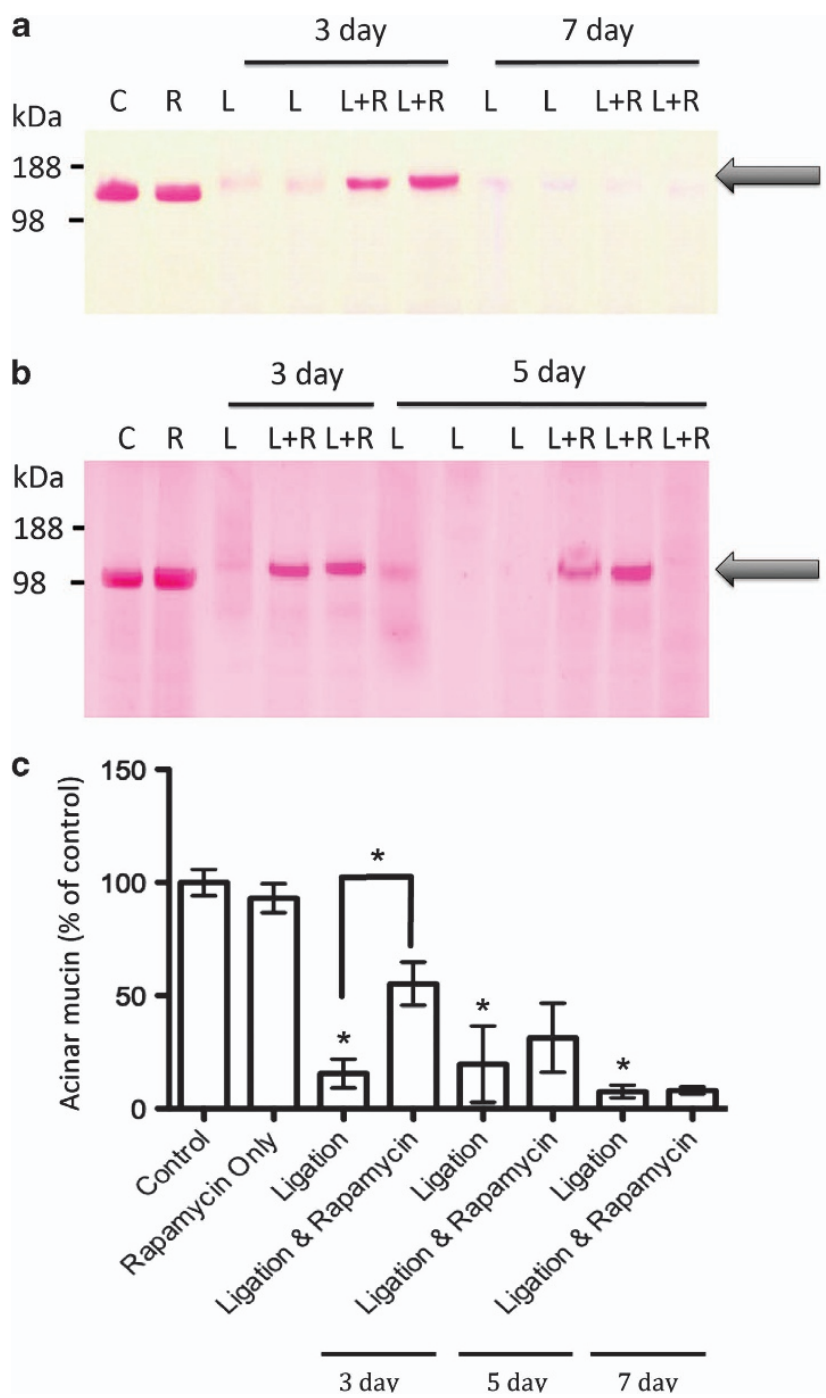

Figure 4 Periodic acid-Schiff's staining of glandular homogenates of 3 and 7 day (a) and 3 and 5 day (b). PAS staining of all ligation only (L) groups marked a significant loss of acinar mucin. The 3 day ligation with rapamycin group $(L+R)$ showed only partial loss of mucin (indicated by arrow), suggesting rapamycin reduced acinar loss of mucin. Five days of rapamycin treatment following ductal ligation revealed transitional results between a partial loss or a complete loss of mucin. No staining was seen after 7 days of rapamycin treatment following ligation. Densiometric analysis of submandibular acinar mucin (c) as a ratio of control $\left({ }^{\star} P<0.05\right)$, shows that increased acinar mucin in 3 days of ligation with rapamycin treatment was significantly higher in comparison with ligation only. The bars represent the mean \pm S.E.M. Data represent results from at least three independent experiments

rapamycin treatment only delays salivary gland atrophy following ductal ligation, as rapamycin is not a full inhibitor of mTOR, owing to the PI3K-negative feedback mechanism (which re-activates $\mathrm{mTORC} 1$ via the TSC $1 / 2$ complex). ${ }^{20}$ It is possible rapamycin is ineffective against the negative feedback mechanism because rapamycin only inhibits mTORC1, ${ }^{21}$ although there has been some evidence to suggest that the prolonged rapamycin treatment inhibits mTORC2 assembly, ${ }^{22}$ which may be relevant to the changes observed from day 3 to day 5 in our study. Using a second generation mTOR inhibitor, Torin1, which is thought to inhibit all kinase-dependent functions of $\mathrm{mTOR},{ }^{23}$ we obtained essentially identical results to rapamycin. It is possible that rapamycin had been effective in mTOR inhibition, yet S6K1 and 4E-BP1 were activated via mTOR-independent phosphorylation of S6K1 and 4E-BP1 (a mechanism suggested by other studies ${ }^{24}$ ).

Evidence from this study leads to the conclusion that inhibition of mTOR can delay ligation-induced atrophy of salivary glands, however only affecting acinar, but not ductal, atrophic processes.

\section{Materials and Methods}

Submandibular duct ligation surgery. A total of 37 adult female ICR mice were obtained from Charles Rivers Laboratories (Margate, UK); weighing an average of 20-25 g upon arrival. On arrival mice were housed in groups of four, with food and water provided ad libitum. A $12 \mathrm{~h}$ light-dark cycle was maintained at a constant temperature of $20-22{ }^{\circ} \mathrm{C}$. Environmental enrichments (tunnels and nesting material) were provided in each cage. Animals were allowed to acclimatize to their new environment for 1 week before experimental procedures. All animal studies and procedures were conducted in accordance with UK Home Office Animal (Scientific Procedures) Act 1986. The mice were weighed and anaesthetized with xylazine $(5 \mathrm{mg} / \mathrm{Kg}) / \mathrm{ketamine}(25 \mathrm{mg} / \mathrm{Kg})$ i.p. injections, and placed on a controlled heating pad to maintain the body temperature. The depth of anesthesia was assessed by pedal reflex. Held in the supine position with the neck extended, a skin incision $\sim 0.5 \mathrm{~cm}$ long was made in the midline of the neck (on the medial side of the angle of the mandible), the fat surrounding the salivary glands was cleared via blunt dissection and subsequently the left submandibular gland duct was isolated. The left submandibular excretory duct was ligated using a 6-0 Ethicon suture (Johnson and Johnson Intl, Brussels, Belgium).

After ligation of the main secretory duct, the neck was sutured. The mice were allowed to recover from anaesthesia in a cage maintained in a warm room and were administered analgeics (buprenorphine, $10 \mu \mathrm{g} / \mathrm{kg}$ ) post surgery. Aseptic conditions were used throughout the surgical procedure of duct ligation to reduce the risk of infection

Submandibular gland samples were collected for analysis. The samples were designated in to groups: the control and experimental groups.

The control groups were either unoperated controls $(n=4)$, receiving drug vehicle for 3 days $(n=4)$ or receiving rapamycin injections for 3 days $(n=4)$.

The experimental groups underwent unilateral submandibular excretory duct ligation surgery under recovery anaesthesia for either $3(n=3), 5(n=3)$ or 7 days $(n=4)$, or they underwent surgery whilst also receiving $5 \mathrm{mg} / \mathrm{kg}$ per day of rapamycin (subcutaneous (s.c.)) for $3(n=4), 5(n=4)$ or 7 days $(n=7)$ post surgery.

At the end of experiments, submandibular glands were removed, weighed and tissues were either fixed in $4 \%$ formalin overnight or snap frozen in liquid nitrogen for biochemical analysis.

Contralateral submandibular glands from the experimental mice were not collected as controls, as they experience compensative hyperplasia when the other gland is extirpated or ligated. ${ }^{25,26}$

Animal body weights were recorded daily. Mice were sacrificed by an overdose of pentobarbitone.

Rapamycin treatment. Rapamycin was resuspended in a stock solution at $20 \mathrm{mg} / \mathrm{ml}$ in DMSO and stored at $-20^{\circ} \mathrm{C}$ until used. For vehicle controls, animals were injected (s.c.) with $200 \mu \mathrm{l}$ of rapamycin injection vehicle (10\% polyethylene glycol 400 and $17 \%$ tween-80). The experimental groups received s.c. injections, each injection consisted of $5 \mathrm{mg} / \mathrm{kg}$ per day of Rapamycin diluted in $200 \mu \mathrm{l}$ of injection vehicle (10\% polyethylene glycol 400 and $17 \%$ tween-80), all as previously demonstrated. ${ }^{27}$ This particular dose was chosen because of the efficacy and comparative effectiveness of the inhibition of mTOR signaling by rapamycin as shown in previous studies, ${ }^{28,29}$ furthermore, in preparatory undertakings for this study, this was found to be the maximum dose without seeing significant body weight loss. No toxicity or adverse effects of the compound were identified in this experiment, in accordance with the previous rapamycin studies. ${ }^{29,30}$

Histochemical staining of tissue samples. Submandibular glands were embedded in wax and $5 \mu \mathrm{m}$ thick sections were cut and mounted on superfrost plus-coated slides. 


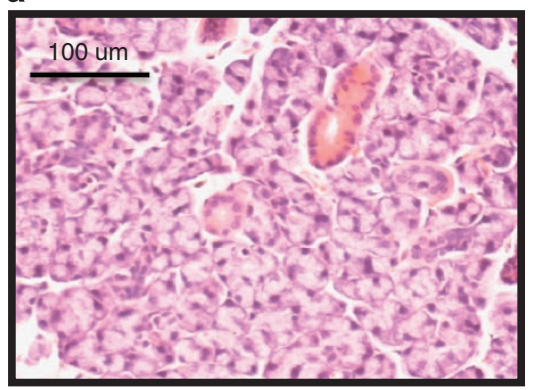

b

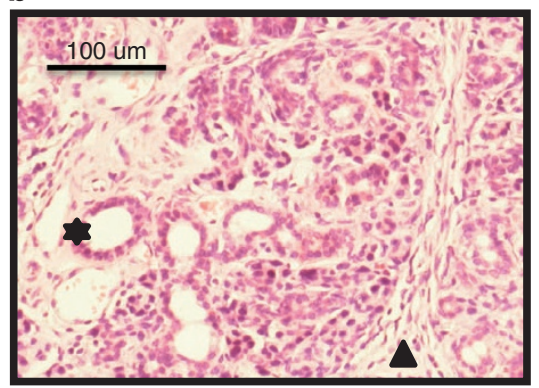

c
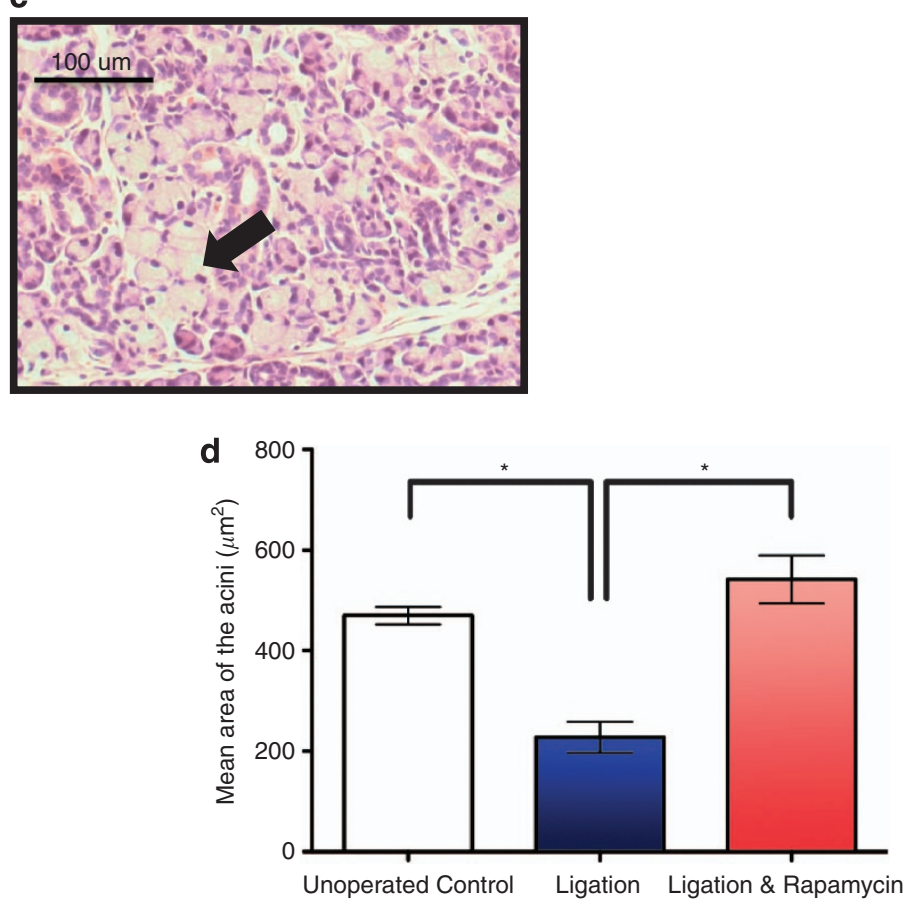

Figure 5 Haematoxylin and Eosin (H\&E) staining of submandibular glands in control (a), following 3 day periods of ligation (b) and ligation with rapamycin treatment (c). The unoperated submandibular gland indicates a conventional appearance of acini and ductal cells. Ligation revealed infiltration of a large number of inflammatory cells (mostly neutrophils and macrophages; arrowhead) and duct luminal dilation (star), exemplary of the atrophic state. Ligation with rapamycin treatment, revealed lack of atrophy in intact acinar cells (arrow). Morphometric analysis of the H\&E-stained samples indicated the mean area of acini (d) from the control, 3 day ligation and 3 day ligation with rapamycin treatment. Ligation significantly decreased the size of the acini ( $\left.{ }^{*} P<0.0001\right)$ in comparison with control. Rapamycin treatment post ligation showed a significant increase in acini area ( $\left.{ }^{*} P<0.0001\right)$. Data is expressed as mean \pm S.E.M.

General morphology of the tissue sections was assessed by H\&E staining. The secretory granules inside acinar cells were identified by AB/PAS staining. Granular ductal kallikreins were stained using DMAB, as previously demonstrated. ${ }^{10}$

Morphometric analysis. From the submandibular gland samples prepared for histochemical staining, 20 acini per sample were randomly selected and the mean area $\left(\mu \mathrm{m}^{2}\right)$ was measured using Leica TCS SP2 confocal microscope software version 2.1 (Leica Microsystems, Heidelberg, Germany).

Tissue preparation and immunoblotting. Tissue specimens stored at $-80^{\circ} \mathrm{C}$ were homogenized in 19 volumes (wt/vol) of ice-cold homogenization buffer ( $1 \%$ Triton X-100, $1 \mathrm{mM}$ EDTA, and a $1 \% \mathrm{v} / \mathrm{v}$ dilution of protease inhibitor cocktail set 1 (Merck Chemicals Ltd, Nottingham, UK) using an Ultra-Thurrax homogenizer (IKA Labortechnik, Staufen, Germany).

SDS-PAGE of samples was carried out (NUPAGE Novex Bis-Tris 4-12\% gel; Life technologies, Paisley, UK). Proteins resolved by electrophoresis were then electroblotted onto $0.45 \mu \mathrm{m}$ nitrocellulose membranes (Anderman and Co., Kingston-Upon-Thames, UK).
The procedure followed for immunoblotting is that which has been previously established. ${ }^{31}$

Membranes were imaged in a ChemiDoc Imaging System (BIORAD Laboratories Ltd, Hertfordshire, UK), with optimized exposure times and the built-in highsensitivity blot detection which highlights over-saturated pixels, to obtain ideal exposure in images of the protein bands.

Band intensity from immunoblots were quantified using the image analysis software ImageJ version $1.46(\mathrm{NIH}$, Maryland, MD, USA), with each bar representing the mean normalized from the ratio of $\beta$-actin \pm S.E.M.

Antibodies. Anti-phospho-4E-BP1 (1:1000 for western blotting), antiphospho-S6 ribosomal protein (1:1000 for western blotting), anti-mTOR $(1: 1000$ for western blotting) were obtained from Cell Signaling Technology (Hertfordshire, UK) and anti- $\beta$-actin was from Sigma-Aldrich (St. Louis, MO, USA).

Secondary antibodies included polyclonal goat anti-mouse immunoglobulin-HRP (P0447) and polyclonal goat anti-rabbit immunoglobulin-HRP (P0448) from Dako Ltd (Ely, UK). 

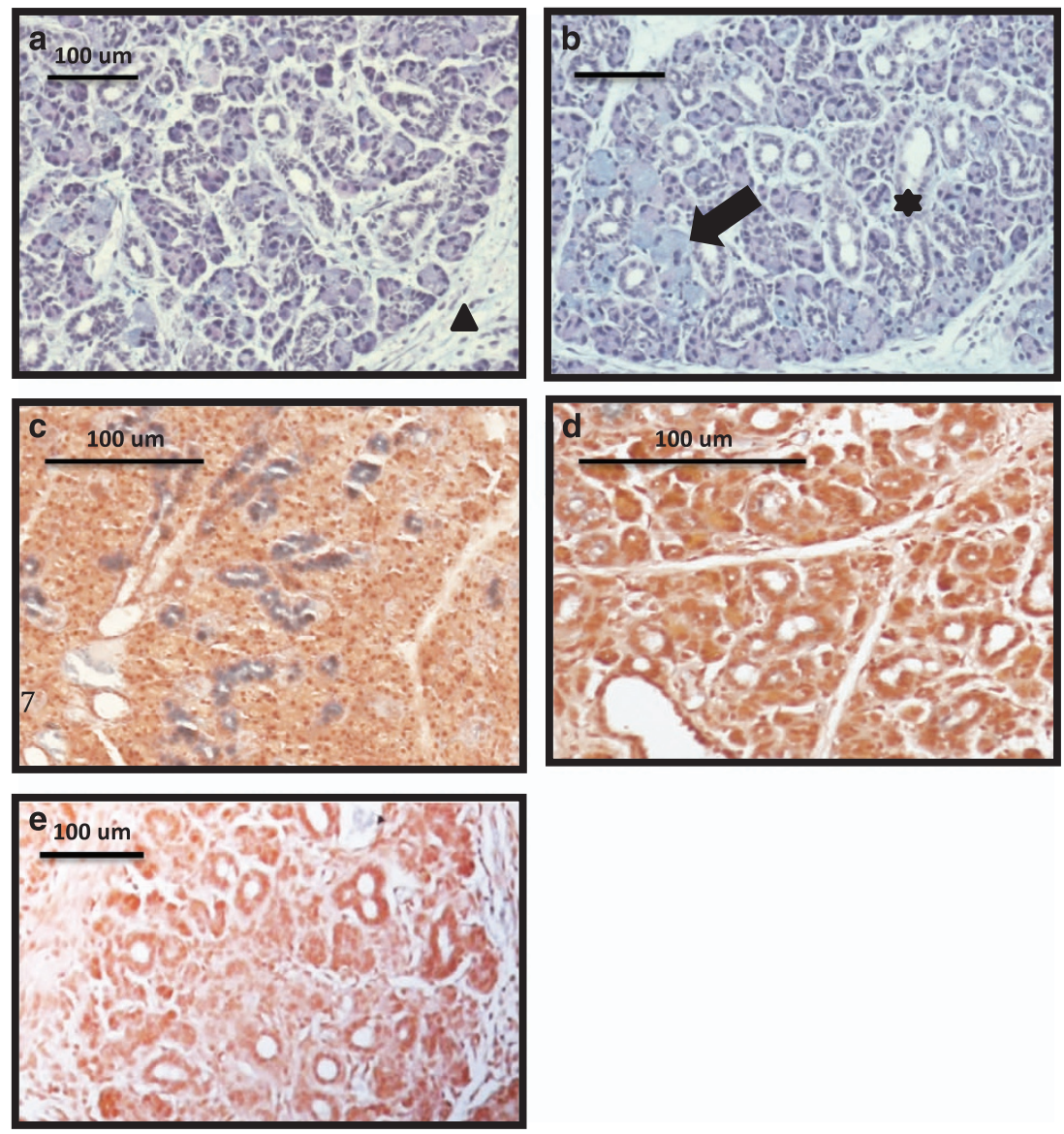

Figure 6 Alcian blue/PAS staining of submandibular glands after 3 days of ligation (a) and ligation with rapamycin treatment (b). The ligated state revealed signs of atrophy such as shrunken acinar cells with no secretory granules, duct luminal dilation and presence of inflammatory cells (mostly neutrophils and macrophages; arrowhead). Rapamycin treatment following ductal ligation, showed intact acinar cells (arrow) and although the ducts displayed larger lumena (star), not all acinar cells appeared enlarged. Histochemical staining of granular ducts by DMAB-nitrite on unoperated controls (c), 3 day ligation (d) and 3 day ligation and rapamycin (e). Lack of blue DMAB on ligation and ligation with rapamycin treatment, shows a loss of blue DMAB staining indicating loss of kallikrein-containing secretory granules in ductal cells.

Periodic acid-Schiff's staining. PAS of glandular homogenates was used to assess glycoproteins. After electrophoreses the gel was fixed in methanol and acetic acid, incubated in $1 \%$ periodic acid for $15 \mathrm{~min}$, rinsed with double distilled water and stained with Schiff's reagent for up to $60 \mathrm{~min}$.

Statistical analysis. Results were expressed as means \pm S.E.M., and were statistically compared by ANOVA followed by student's $t$-test; $P<0.05$ was considered statistically significant.

\section{Conflict of Interest}

The authors declare no conflict of interest.

Acknowledgements. We gratefully acknowledge the support of the Newland Pedley Studentship in Dental Research. We would also like to acknowledge Adelaide Annan for guidance on histological techniques.

1. Jemal A, Siegel R, Ward E, Hao Y, Xu J, Thun MJ. Cancer statistics, 2009. CA Cancer J Clin 2009; 59: 225-249.

2. Nagler RM, Baum BJ. Prophylactic treatment reduces the severity of xerostomia following radiation therapy for oral cavity cancer. Arch Otolaryngol Head Neck Surg 2003; 129: 247-250.

3. Vissink A, Jansma J, Spijkervet FKL, Burlage FR, Coppes RP. Oral sequelae of head and neck radiotherapy. Crit Rev Oral Biol Med 2003; 14: 199-212.

4. Cummins M, Dardick I, Brown D, Burford-Mason A. Obstructive sialadenitis: a rat model. J Otolaryngol 1994; 23: 50-56.
5. Takahashi S, Nakamura S, Suzuki R, Islam N, Domon T, Yamamoto T et al. Apoptosis and mitosis of parenchymal cells in the duct-ligated rat submandibular gland. Tissue Cell 2000; 32: 457-463.

6. Harrison JD, Fouad HM, Garrett JR. The effects of ductal obstruction on the acinar cells of the parotid of cat. Arch Oral Biol 2000; 45: 945-949.

7. Mieulet V, Roceri M, Espeillac C, Sotiropoulos A, Ohanna M, Oorschot V et al. S6 kinase inactivation impairs growth and translational target phosphorylation in muscle cells maintaining proper regulation of protein turnover. Am J Physiol Cell Physiol 2007; 293: C712-C722.

8. Ma XM, Blenis J. Molecular mechanisms of mTOR-mediated translational control. Nat Rev Mol Cell Biol 2009; 10: 307-318.

9. Herbert TP, Tee AR, Proud CG. The extracellular signal-regulated kinase pathway regulates the phosphorylation of $4 \mathrm{E}-\mathrm{BP} 1$ at multiple sites. $J$ biol chem 2002; 277 : 11591-11596.

10. Silver N, Proctor GB, Arno M, Carpenter GH. Activation of mTOR coincides with autophagy during ligation-induced atrophy in the rat submandibular gland. Cell Death Dis 2010; 1: e14.

11. Correia PN, Carpenter GH, Osailan SM, Paterson KL, Proctor GB. Acute salivary gland hypofunction in the duct ligation model in the absence of inflammation. Oral Dis 2008; 14: 520-528.

12. Cotroneo E, Proctor GB, Paterson $\mathrm{KL}$, Carpenter GH. Early markers of regeneration following ductal ligation in rat submandibular gland. Cell Tissue Res 2008; 332: 227-235.

13. Gresik EW. Postnatal developmental changes in submandibular glands of rats and mice. J Histochem Cytochem 1980; 28: 860-870.

14. Takahashi S, Shinzato K, Nakamura S, Domon T, Yamamoto T, Wakita M. Cell death and cell proliferation in the regeneration of atrophied rat submandibular glands after duct ligation. J Oral Pathol Med 2004; 33: 23-29.

15. Carpenter GH, Osailan SM, Correia P, Paterson KP, Proctor GB. Rat salivary gland ligation causes reversible secretory hypofunction. Acta Physiol (Oxf) 2007; 189: 241-249. 
16. Shori DK, Proctor GB, Garrett JR, Zhang XS, Carpenter GH. Histochemical staining of ducts in submandibular glands by DMAB-nitrite detects stored tissue kallikreins. Biochem Soc Trans 1997; 25: 28S.

17. Cotroneo E, Proctor GB, Carpenter GH. Regeneration of acinar cells following ligation of rat submandibular gland retraces the embryonic-perinatal pathway of cytodifferentiation. Differentiation 2010; 79: 120-130.

18. Burnett PE, Barrow RK, Cohen NA, Snyder SH, Sabatini DM. RAFT1 phosphorylation of the translational regulators p70 S6 kinase and 4E-BP1. Proc Natl Acad Sci USA 1998; 95(4): 1432-1437.

19. Isotani S, Hara K, Tokunaga C, Inoue H, Avruch J, Yonezawa K. Immunopurified mammalian target of rapamycin phosphorylates and activates p70 S6 kinase alpha in vitro. J biol chem 1999; 274: 34493-34498.

20. Guertin DA, Sabatini DM. The pharmacology of mTOR inhibition. Sci Signal 2009; 2: pe24.

21. Zaytseva YY, Valentino JD, Gulhati P, Evers BM. mTOR inhibitors in cancer therapy. Cancer Lett 2012; 319: 1-7.

22. Sarbassov DD, Ali SM, Sengupta S, Sheen J-H, Hsu PP, Bagley AF et al. Prolonged rapamycin treatment inhibits mTORC2 assembly and Akt/PKB. Mol Cell 2006; 22: 159-168.

23. Thoreen CC, Kang SA, Chang JW, Liu Q, Zhang J, Gao Y et al. An ATP-competitive mammalian target of rapamycin inhibitor reveals rapamycin-resistant functions of mTORC1. J biol chem 2009; 284: 8023-8032.

24. Liu Y, Vertommen D, Rider MH, Lai Y-C. Mammalian target of rapamycin-independent $\mathrm{S} 6 \mathrm{~K} 1$ and $4 \mathrm{E}-\mathrm{BP} 1$ phosphorylation during contraction in rat skeletal muscle. Cell Signal 2013; 25: 1877-1886.

25. Walker NI, Gobe GC. Cell death and cell proliferation during atrophy of the rat parotid gland induced by duct obstruction. J pathol 1987; 153: 333-344.
26. Schwartz-Arad D, Michaeli Y, Zajicek G. Compensatory hyperplasia of the rat submandibular gland following unilateral extirpation. J Dent Res 1991; 70: 1328-1331.

27. Liu KJ, Arron JR, Stankunas K, Crabtree GR, Longaker MT. Chemical rescue of cleft palate and midline defects in conditional GSK-3beta mice. Nature 2007; 446: 79-82.

28. Hu M, Ekshyyan O, Herman Ferdinandez L, Rong X, Caldito G, Nathan C-AO. Efficacy and comparative effectiveness of sirolimus as an anticancer drug. Laryngoscope 2011; 121 978-982.

29. Shillingford JM, Piontek KB, Germino GG, Weimbs T. Rapamycin ameliorates PKD resulting from conditional inactivation of Pkd1. J Am Soc Nephrol 2010; 21: 489-497.

30. Banerjee S, Gianino SM, Gao F, Christians U, Gutmann DH. Interpreting mammalian target of rapamycin and cell growth inhibition in a genetically engineered mouse model of Nf1-deficient astrocytes. Mol Cancer Ther 2011; 10: 279-291.

31. Carpenter GH, Proctor GB, Ebersole LE, Garrett JR. Secretion of IgA by rat parotid and submandibular cells in response to autonomimetic stimulation in vitro. Int Immunopharmacol 2004; 4: 1005-1014.

(c) (1) (2) Cell Death and Disease is an open-access journal published by Nature Publishing Group. This work is licensed under a Creative Commons Attribution-NonCommercialShareAlike 3.0 Unported License. To view a copy of this license, visit http://creativecommons.org/licenses/by-nc-sa/3.0/ 